\title{
Management of giant prolactinoma causing craniocervical instability: illustrative case
}

\author{
Arunit J. S. Chugh, MD, ${ }^{1}$ Mohit Patel, MD, ${ }^{1}$ Lorayne Chua, MD, ${ }^{2}$ Baha Arafah, MD, ${ }^{2}$ Nicholas C. Bambakidis, MD, ${ }^{1}$ and \\ Abhishek Ray, MD ${ }^{1}$
}

Departments of ${ }^{1}$ Neurological Surgery and ${ }^{2}$ Endocrinology, Case Western Reserve University, University Hospitals Cleveland Medical Center, Cleveland, Ohio

BACKGROUND Giant prolactinomas $(>4 \mathrm{~cm})$ are a rare entity, constituting less than $1 \%$ of all pituitary tumors. Diagnosis can usually be achieved through endocrinological analysis, but biopsy may be considered when trying to differentiate between invasive nonfunctioning pituitary adenomas and primary clival tumors such as chordomas.

OBSERVATIONS The authors presented a rare case of a giant prolactinoma causing significant clival and occipital condyle erosion, which led to craniocervical instability. They provided a review of the multimodal management. Management involved medical therapy with dopamine agonists, and surgery was reserved for acute neural compression or dopamine agonist resistance, with the caveat that surgery was extremely unlikely to lead to normalization of serum prolactin in dopamine agonist-resistant tumors.

LESSONS Adjunctive surgical therapy may be necessary in cases of skull base erosion, particularly when erosion or pathological fractures involve the occipital condyles. Modern posterior occipital-cervical fusion techniques have high rates of arthrodesis and can lead to symptomatic improvement. This procedure should be considered early in the multimodal approach to giant prolactinomas because of the often dramatic response to medical therapy and potential for further craniocervical instability.

https://thejns.org/doi/abs/10.3171/CASE2158

KEYWORDS dopamine agonists; giant prolactinomas; occipital-cervical fusion; occipital-cervical instability

Pituitary adenomas are one of the most common intracranial tumors, with a prevalence of approximately $20 \%$ on autopsy and radiographic studies. ${ }^{1}$ Prolactinomas represent the most common form of functioning pituitary adenomas. These lesions can present as micro- $(<1 \mathrm{~cm})$ or macroadenomas $(>1 \mathrm{~cm})$, and patients can present with various symptoms, including galactorrhea, decreased libido, and gynecomastia. ${ }^{2,3}$ Giant prolactinomas $(>4 \mathrm{~cm})$ are a rare subtype that account for $0.5 \%$ to $2 \%$ of all pituitary tumors. ${ }^{2,3}$ In even rarer cases, these invasive tumors can cause significant skull base erosion and lead to craniocervical instability. We found only four such cases reported in the literature dating back to 1979. We present a rare case of a giant prolactinoma causing occipital-cervical (OC) instability through bony destruction of the occipital condyles and review the literature on this topic.

\section{Illustrative Case}

\section{History and Physical}

The patient was a 39-year-old man with a previous medical history significant for testicular cancer that required unilateral orchiectomy but no chemotherapy or radiation treatment. He presented with headache and neck pain of several weeks' duration. He described intermittent shooting pain to his shoulders and occiput that was exacerbated with neck flexion or extension. The patient reported lack of libido but denied having galactorrhea, rhinorrhea, or changes in vision. The clinical exam did not reveal demonstrable abnormalities in visual acuity, visual fields testing, or cranial nerve function. Furthermore, no gynecomastia or galactorrhea was detected, nor were there clinical signs of additional pituitary hormone abnormalities.

ABBREVIATIONS CSF = cerebrospinal fluid; $\mathrm{CT}=$ computed tomography; $\mathrm{MRI}=$ magnetic resonance imaging; $\mathrm{OC}=$ occipital-cervical; $\mathrm{PRL}=$ prolactin; $\mathrm{VA}=$ vertebral artery . INCLUDE WHEN CITING Published June 7, 2021; DOI: 10.3171/CASE2158.

SUBMITTED January 25, 2021. ACCEPTED February 25, 2021.

(C) 2021 The authors, CC BY-NC-ND 4.0 (http://creativecommons.org/licenses/by-nc-nd/4.0/). 

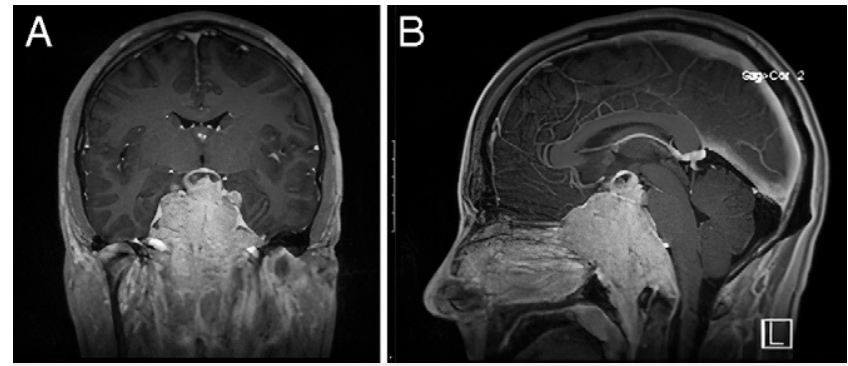

FIG. 1. Coronal (A) and sagittal (B) views of pretreatment MRI. T1-weighted imaging with contrast sequences demonstrating large enhancing mass with involvement of the sellar, suprasellar, and clival regions.

\section{Radiographic and Clinical Workup}

Magnetic resonance imaging (MRI) of the brain showed a large enhancing lesion with extensive skull base involvement that invaded the sella, clivus, sphenoid sinus, and bilateral occipital condyles (Fig. 1). Computed tomography (CT) demonstrated significant erosion of the clivus and occipital condyles (Fig. 2). Dynamic flexion/ extension radiographs were not obtained for several reasons. Given the high radiographic suspicion for craniocervical instability based on prior imaging and the patient's mechanical neck pain that worsened with flexion and extension, we did not believe that flexion/extension radiographs would add more diagnostic utility. In addition, diagnosing craniocervical instability on the basis of plain radiographs can be difficult, especially in the setting of existing significant skull base erosion.

Laboratory tests were notable for a serum prolactin (PRL) level of 22,349 $\mu \mathrm{g} / \mathrm{L}$, follicle-stimulating hormone level of $16 \mathrm{IU} / \mathrm{L}$, luteinizing hormone level of $5.7 \mathrm{IU} / \mathrm{L}$, and testosterone level of $<30 \mu \mathrm{g} / \mathrm{dL}$. These biochemical studies were consistent with a prolactinoma and central hypogonadism. The rest of the pituitary panel was within normal limits, suggesting no evidence of central adrenal insufficiency, diabetes insipidus, or central hypothyroidism.

\section{Management}

Given the symptomatic nature of the mass and craniocervical instability, surgical stabilization was recommended. Because there was only disruption of the occipital condyles without obvious ligamentous injury (based on careful review of MRI), the decision was made to place the patient in a collar instead of a halo before surgery. One week after the diagnosis, the patient ultimately underwent
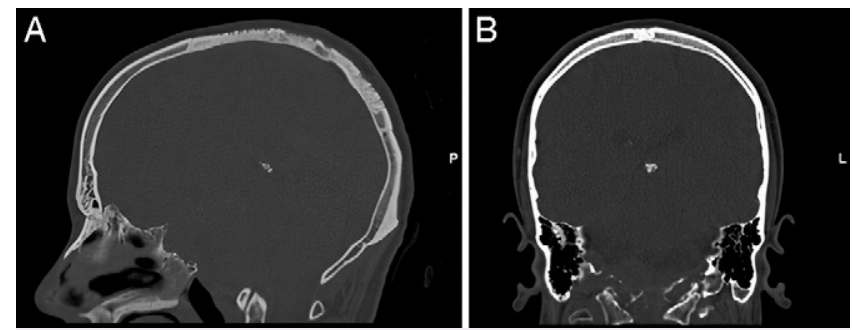

FIG. 2. Sagittal (A) and coronal (B) views of pretreatment CT. Noncontrast sequences demonstrating significant erosion of the skull base with near complete replacement of the bilateral occipital condyles.

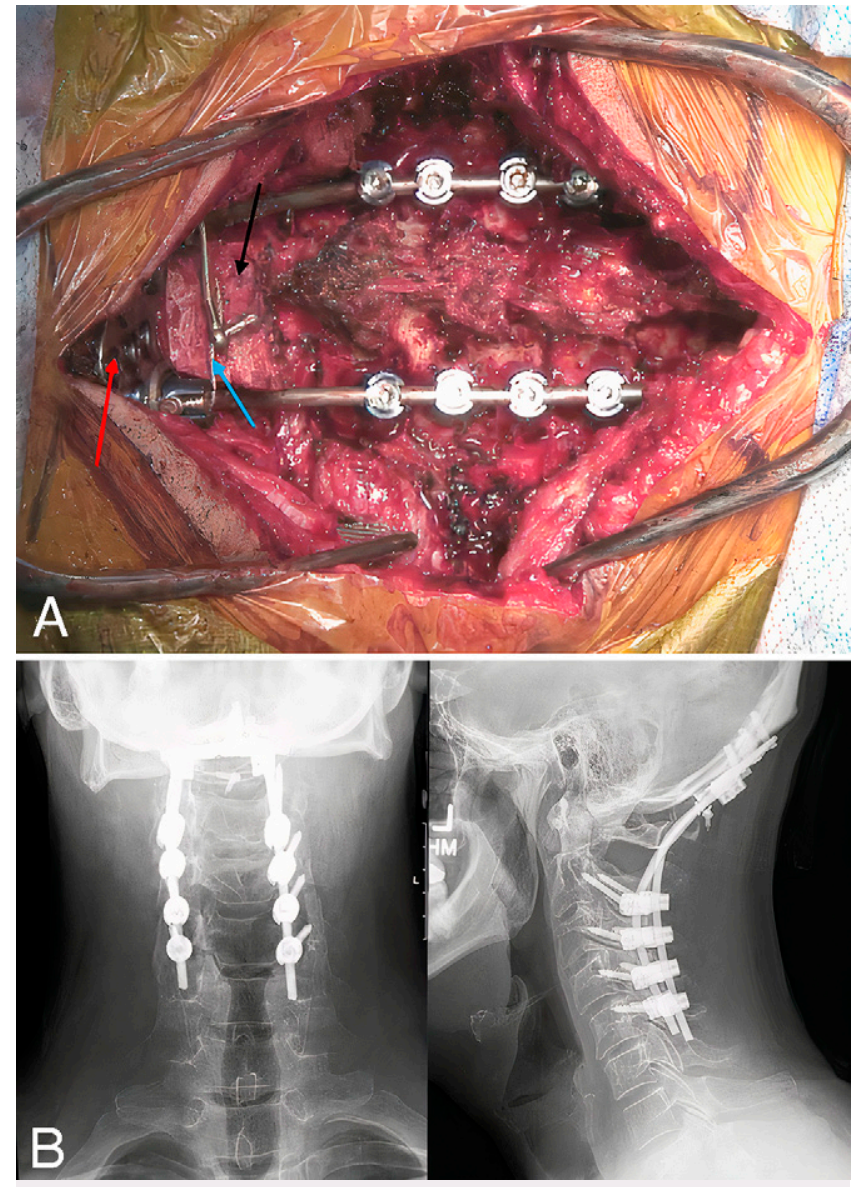

FIG. 3. Intraoperative photograph (A) and postoperative upright radiographs (B). Red arrow indicates occipital plate; blue arrow indicates Songer cables; black arrow indicates iliac crest autograft.

occiput-to-C5 instrumentation and fusion with iliac crest tricortical autograft that was fixed at the OC junction using Songer cables (Fig. 3). Intraoperative spinal navigation was used to optimize the position of the occipital plate and C2 pars screws. The patient was initially started on cabergoline at $0.25 \mathrm{mg}$ daily, with a gradual increase of the dose to $0.5 \mathrm{mg}$ daily. Four months later, MRI of the patient's brain showed a marked reduction in the size of the mass (Fig. 4), and the PRL level was decreased to $3,900 \mu \mathrm{g} / \mathrm{L}$. Follow-up cervical spinal radiographs demonstrated appropriate sagittal alignment clinically and radiographically. Although the patient had a limited range of motion as expected, he had full resolution of his neck pain. At the 1-year mark, the patient was clinically doing well and had plans to undergo cranial and cervical spine imaging to evaluate for bony fusion. His PRL level had decreased to $1,576 \mu \mathrm{g} / \mathrm{L}$ at the 1-year follow-up as well. $\mathrm{He}$ is currently on $1 \mathrm{mg}$ of cabergoline and $20 \mathrm{mg}$ of tamoxifen daily.

\section{Discussion}

\section{Observations}

Radiographically, giant prolactinomas can be difficult to distinguish from primary clival tumors, including chordomas. There are reports of adenomas with clival invasion being misdiagnosed as 


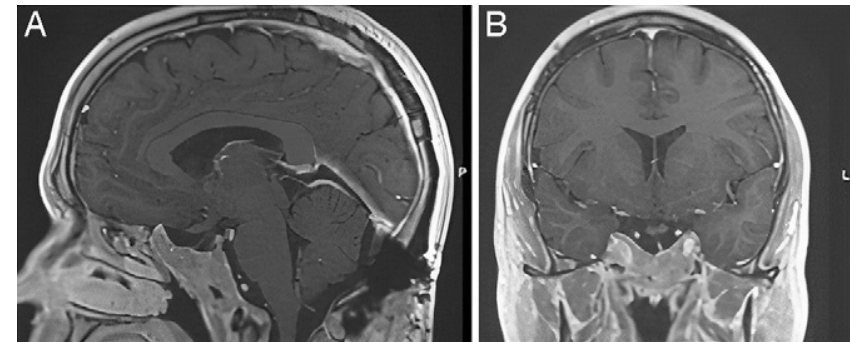

FIG. 4. Sagittal (A) and coronal (B) views of posttreatment MRI. T1-weighted imaging with contrast sequences demonstrating significant reduction in the size of the tumor.

chordomas. ${ }^{4}$ Chordomas tend to be T2 hyperintense on MRI, whereas pituitary adenomas are largely isointense, ${ }^{5}$ which was consistent with this patient. It is important to consider invasive pituitary adenomas in the differential diagnosis for clival masses and include pituitary function studies in the evaluation.

Invasive prolactinomas typically have markedly elevated $\mathrm{PRL}$ levels; therefore, a biopsy is usually not warranted in cases exhibiting this effect. Serial dilutions should be done in suspected cases in which PRL is mildly elevated to rule out the "hook effect." PRL levels $>250 \mu \mathrm{g} / \mathrm{L}$ should raise suspicion for a prolactinoma, with values $>1,000 \mu \mathrm{g} / \mathrm{L}$ being highly suggestive. ${ }^{3,6}$ If laboratory studies are not diagnostic, then biopsy is warranted and usually performed in an endonasal fashion.

Given the well-substantiated response to medical therapy, resection is usually not necessary unless tumors are refractory to medical therapy. ${ }^{6,7}$ Yang and colleagues reported on the outcomes of 36 patients with invasive prolactinomas treated with bromocriptine with an average 44-month follow-up. ${ }^{8}$ They found that only 5 of these patients $(14 \%)$ required surgical debulking. In the surgical group, normalization of serum PRL was never achieved with surgery alone. They concluded that patients who attained more than a $25 \%$ reduction in tumor volume and normal serum PRL with 3 months of bromocriptine therapy had a high possibility of showing a good longterm response. For patients who had a good reduction in tumor volume but serum PRL that did not normalize, high doses of bromocriptine were advocated. Shimon et al. described the outcomes of 18 patients with giant prolactinomas larger than $60 \mathrm{~mm} .{ }^{9}$ Of the 14 patients who had preoperative visual disturbances, 12 had improvement with medical therapy. Nine patients had surgery with elevated $\mathrm{PRL}$; however, none of those patients had remission postoperatively. In another recent study, 13 of 27 patients initially treated with bromocriptine had PRL normalization, whereas none of the 14 patients with first-line surgery had that result. ${ }^{10}$

Rates of new anterior pituitary insufficiency are especially high after surgery for giant pituitary adenomas. In one recent study describing the surgical outcomes of 50 giant pituitary tumors, $22 \%$ and $14 \%$ of cases had one axis or more than one axis affected, respectively, with $38 \%$ of cases having total removal, $18 \%$ near-total removal, and $44 \%$ subtotal removal. ${ }^{11}$ Hypopituitarism is more common after surgery than after medical treatment for giant prolactinomas. Hamidi and colleagues reported 71 patients with giant prolactinomas, 70 of whom received dopamine agonist therapy, 30 of whom underwent surgery, and 10 of whom received radiation. ${ }^{12}$ Rates of adrenal insufficiency, hypothyroidism, growth hormone deficiency, and diabetes insipidus were all significantly higher in the surgery group. They also found that therapeutic response did not correlate with sex, age, initial PRL, tumor size, or modality of therapy. Radiation is an option, but visual deterioration may be significant, and long-term visual outcome is poorly reported. In one study of 59 patients who underwent surgery followed by stereotactic radiosurgery for functioning macroadenomas and giant adenomas, 7 patients had visual deterioration. ${ }^{13}$

Hence, first-line treatment with dopamine agonists should be considered, with surgery reserved for acute compression symptoms and dopamine resistance. With dopamine-resistant invasive prolactinomas, PRL normalization with surgery is unlikely. For these patients, another potential medical option is the use of the well-known alkylating agent temozolomide. ${ }^{14}$ This drug has been used for the treatment of some brain tumors, including glioblastoma multiforme. Attempts to use temozolomide to treat this pathology started in 2006 and have expanded ever since, such that it is now considered a reasonable option to manage invasive/aggressive functioning as well as nonfunctioning pituitary adenomas, including prolactinomas, particularly those that are resistant to dopamine agonist therapy. The drug is reasonably well tolerated by patients. It is not cell cycle dependent, and it crosses the blood-brain barrier. The response rate as demonstrated by biochemical studies (e.g., lowering serum $\mathrm{PRL}$ ) or imaging studies is nearly $65 \%$ to $70 \%{ }^{14}$ The drug will be a reasonable option for our patient if he becomes resistant to dopamine agonist therapy. Although the drug offers a new therapeutic option for patients with invasive tumors, questions concerning continuous versus intermittent therapy, duration of treatment, and potential long-term side effects of using an alkylating agent have yet to be answered.

\section{Lessons}

Our patient had a profound response to cabergoline, with a dramatic reduction in the size of the tumor burden. On postoperative MRI (Fig. 4), tumor volume decreased substantially after 3 months of dopamine agonist therapy. Given the overall steady improvement, the dose was sequentially increased to $1 \mathrm{mg}$ cabergoline daily at the 12-month follow-up visit. It is highly likely that further decline in serum PRL levels may decelerate. Along the same lines, residual tumor size may not change dramatically over time.

Agonist therapy and rapid tumor shrinkage have their own risks, including cerebrospinal fluid (CSF) fistulas, pneumocephalus, and optic chiasm herniation. ${ }^{10,15}$ Adjunctive surgical fixation may be required to manage cases of recurrent sinusitis, CSF leak, or, as in this case, craniocervical instability. Rarely, bony erosion of the skull base at the level of the craniocervical junction from an invasive prolactinoma can lead to instability.

A literature review of prior cases of craniocervical instability secondary to giant prolactinomas was performed. A search strategy using controlled vocabulary in combination with keywords was performed via the following electronic databases: MEDLINE via PubMed, Ovid, Embase via Embase.com, the Cochrane Library (Wiley interface, current issue), Web of Science/Knowledge via Clarivate Analytics, ClinicalTrials.gov, and SCOPUS. The search was limited to the English-language literature. The references of the searched literature were used to expand the search and include all gray literature. Upon our review, we found only four case reports describing this phenomenon, all of which included patients who presented with pathological fractures that required craniocervical 
TABLE 1. Cases of craniocervical instability secondary to giant prolactinomas

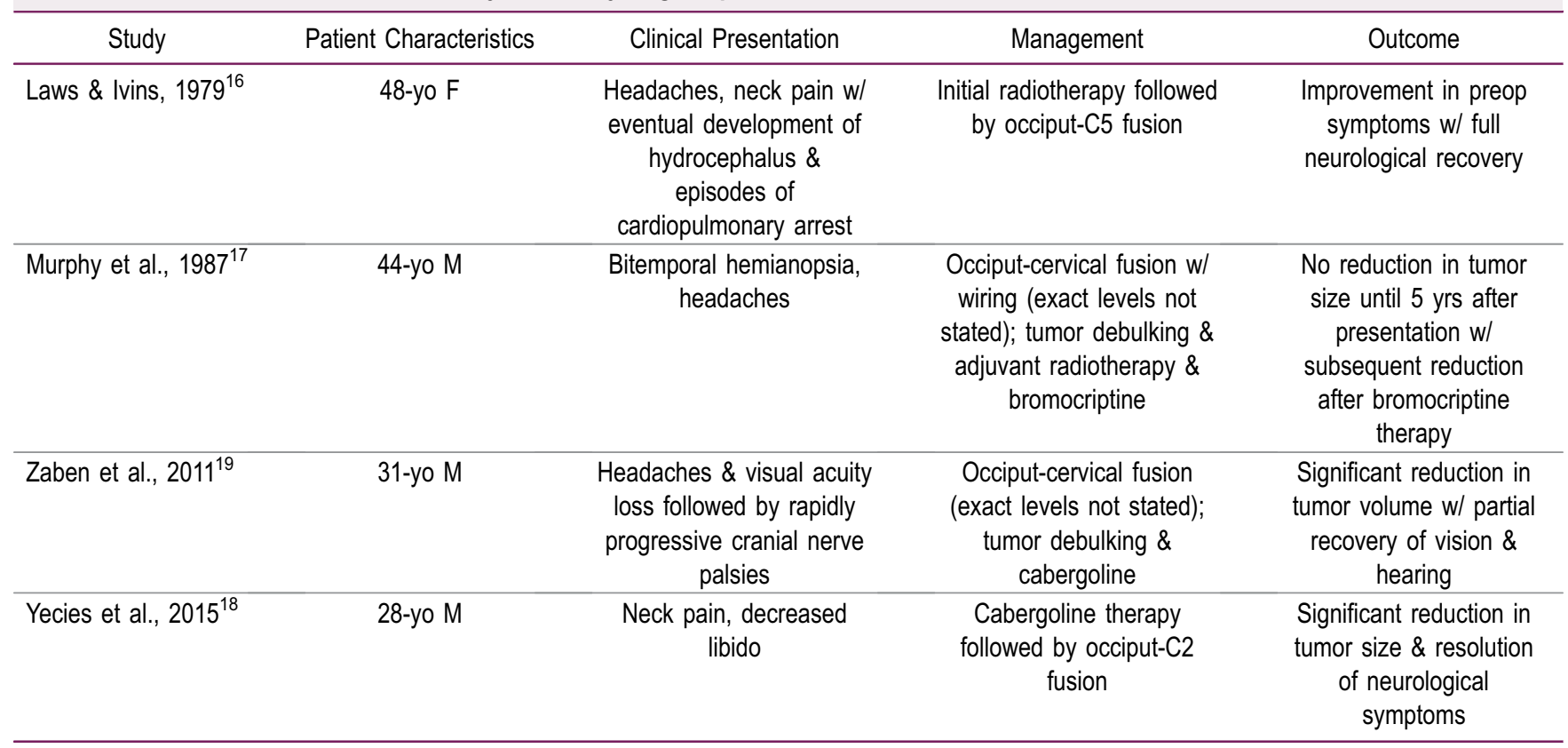

yo $=$ year old

stabilization. ${ }^{16-19}$ Laws and Ivins in 1979 and Zaben et al. in 2011 described patients with giant prolactinomas who became severely symptomatic from brainstem compression. ${ }^{16,19}$ Murphy et al. in 1987 reported a patient who presented with neck pain and bitemporal hemianopsia. ${ }^{17}$ Yecies et al. in 2015 described a patient with neck pain and decreased libido (Table 1). ${ }^{18}$ These cases highlight not only the varying symptomatology with which such patients can present but also the potentially devastating effects that craniocervical instability can have if left untreated.

We believe that when there is bony erosion of the occipital condyles, it is important to treat the $O C$ instability in a relatively urgent fashion because giant prolactinomas can respond more dramatically than smaller macroprolactinomas with dopamine agonist therapy, ${ }^{3}$ in terms of both rate and quantity of tumor volume reduction. However, in the setting of tumor destruction of occipital condyles and lack of any significant bony support, craniocervical instability may worsen with rapid shrinkage of the tumor if concomitant surgical stabilization is not performed. Unlike with atlanto-occipital trauma, good classification systems and methodologies to radiographically diagnose craniocervical instability in the setting of bony erosion by tumor are not available. In fact, classically defined parameters such as basion-axial interval and basion-dental interval, atlanto-occipital interval, Powers ratio, Dublin measure, and $X$-line method may all be normal before dopamine agonist therapy and tumor volume reduction.

OC fusion has undergone significant improvement over the past several decades, and modern fixation devices, including 0-arm navigation techniques, allow for high fusion rates, reaching $100 \%$ in some studies. ${ }^{10,20}$ Often, we use intraoperative spinal navigation to optimize location and maximize screw length for occipital plate placement because hardware failure can occur there. Common indications for $\mathrm{OC}$ fusion include infections, autoimmune disorders (i.e., rheumatoid arthritis), neoplasm, and trauma. The extent of fusion depends on the pathology but normally involves a construct that extends at least from the occiput to C2. Data are limited for occiput$\mathrm{C} 1$ instrumentation alone and are rarely used, partly because most of the biomechanical data arise from trauma literature, in which there are high rates $(>50 \%)$ of associated atlanto-axial injury along with $\mathrm{OC}$ instability. ${ }^{21}$ The decision to extend fusion subaxially is case dependent, and biomechanical studies support increased stability without significantly reducing range of motion. ${ }^{22}$ Some studies have noted an increased risk of subaxial deformity with occiput-C2 fusions, particularly when preoperative subaxial subluxation is present or significant anteversion of the occipital bone against the axis exists. $^{22,23}$

Preoperative planning with CT angiography is key for evaluation of vertebral artery (VA) anatomy and overall bony anatomy. This approach is especially important when it comes to placement of C1-2 transarticular screws, which have a relatively higher risk of VA injury, with rates ranging from $2 \%$ to $4 \% .{ }^{23}$ Other upper cervical instrumentation can include $\mathrm{C} 1$ lateral mass screws combined with $\mathrm{C} 2$ pars or pedicle screws and an occipital plate; this construct has shown similar biomechanical strength as the C1-2 transarticular construct. ${ }^{24}$ Another option is placement of $\mathrm{C} 2$ translaminar screws, which allows for a significantly lower risk of VA injury but an increased risk of spinal cord injury. However, biomechanical strength studies have shown inferiority. ${ }^{25}$ As regards occipital instrumentation, midline plates with screw instrumentation in the keel have been shown to be superior in terms of pullout strength and resistance to cranial settling compared to those with wiring techniques. ${ }^{26}$

Another important aspect in planning is cranial stabilization (i.e., halo) before flipping and positioning, which is especially important in traumatic cases in which multilevel ligamentous damage may be present. In our case, there was only disruption of the occipital condyles without obvious ligamentous injury; hence, a decision was made to place the patient in a collar instead of a halo before 
surgical instrumentation. With regard to arthrodesis and use of graft material, data on the use of autograft versus allograft are limited, but both approaches appear to be equally effective. In a literature review comparing OC fusion techniques, ${ }^{27}$ most surgeons $(97 \%)$ used autograft with relatively high fusion rates, which is consistent across the recent literature. ${ }^{28}$ Adverse events, including vascular injury, spinal cord injury, CSF leak, pseudoarthrosis, dysphagia, and postoperative respiratory compromise, range from $5 \%$ to $11 \%$. Arthrodesis rates are reported to be lower in tumor cases, presumably because of the osteolytic nature of most neoplasms. However, given the relatively high rates of posterior fusion regardless of technique or graft type, significant improvement in neurological signs and symptoms, and overall high patient satisfaction scores (particularly with plate/screw constructs), posterior OC fusion remains an excellent approach for craniocervical stabilization.

In conclusion, giant prolactinomas $(>4 \mathrm{~cm})$ are a rare entity, constituting less than $1 \%$ of all pituitary tumors. Diagnosis can usually be achieved through endocrinological analysis, and biopsy should only be performed when trying to differentiate between invasive nonfunctioning pituitary adenomas and primary clival tumors, such as chordomas. Management involves medical therapy with dopamine agonists, and surgery is reserved for acute neural compression or dopamine agonist resistance, with the caveat that surgery is extremely unlikely to lead to the normal ization of serum PRL in dopamine agonist-resistant tumors. Adjunctive surgical therapy may be necessary in cases of skull base erosion, particularly when erosion or pathological fractures involve the occipital condyles. Modern posterior OC fusion techniques have high rates of arthrodesis and can lead to symptomatic improvement. This procedure should be considered early in the multimodal approach to giant prolactinomas because of the often dramatic response to medical therapy and potential for further craniocervical instability.

\section{References}

1. Ezzat S, Asa SL, Couldwell WT, et al. The prevalence of pituitary adenomas: a systematic review. Cancer. 2004;101(3): 613-619.

2. Ciccarelli A, Daly AF, Beckers A. The epidemiology of prolactinomas. Pituitary. 2005;8(1):3-6.

3. Shrivastava RK, Arginteanu MS, King WA, Post KD. Giant prolactinomas: clinical management and long-term follow up. J Neurosurg. 2002;97(2):299-306.

4. Wong K, Raisanen J, Taylor SL, et al. Pituitary adenoma as an unsuspected clival tumor. Am J Surg Pathol. 1995;19(8):900-903.

5. Pisaneschi M, Kapoor G. Imaging the sella and parasellar region. Neuroimaging Clin N Am. 2005;15(1):203-219.

6. Acharya SV, Gopal RA, Menon PS, et al. Giant prolactinoma and effectiveness of medical management. Endocr Pract. 2010;16(1): 42-46.

7. Cho EH, Lee SA, Chung JY, et al. Efficacy and safety of cabergoline as first line treatment for invasive giant prolactinoma. J Korean Med Sci. 2009;24(5):874-878.

8. Yang MS, Hong JW, Lee SK, et al. Clinical management and outcome of 36 invasive prolactinomas treated with dopamine agonist. J Neurooncol. 2011;104(1):195-204.

9. Shimon I, Sosa E, Mendoza V, et al. Giant prolactinomas larger than $60 \mathrm{~mm}$ in size: a cohort of massive and aggressive prolactin-secreting pituitary adenomas. Pituitary. 2016;19(4): $429-436$
10. Machicado JD, Varghese JM, Orlander PR. Cabergoline-induced pneumocephalus in a medically treated macroprolactinoma. J Clin Endocrinol Metab. 2012;97(10):3412-3413.

11. Gondim JA, Almeida JPC, Albuquerque LAF, et al. Giant pituitary adenomas: surgical outcomes of 50 cases operated on by the endonasal endoscopic approach. World Neurosurg. 2014;82(1-2): e281-e290.

12. Hamidi O, Van Gompel J, Gruber L, et al. Management and outcomes of giant prolactinoma: a series of 71 patients. Endocr Pract. 2019;25(4):340-352.

13. Fu P, He YS, Cen YC, et al. Microneurosurgery and subsequent Gamma Knife radiosurgery for functioning pituitary macroadenomas or giant adenomas: one institution's experience. Clin Neurol Neurosurg. 2016;145:8-13.

14. Syro LV, Rotondo F, Camargo M, et al. Temozolomide and pituitary tumors: current understanding, unresolved issues, and future directions. Front Endocrinol (Lausanne). 2018;9:318.

15. Jones SE, James RA, Hall K, Kendall-Taylor P. Optic chiasmal herniation-an under recognized complication of dopamine agonist therapy for macroprolactinoma. Clin Endocrinol (Oxf). 2000; 53(4):529-534.

16. Laws ER Jr, Ivins JC. Atlanto-occipital instability as a result of pituitary adenoma: case report with successful management by cervicooccipital fusion. Johns Hopkins Med J. 1979;145(3):136-138.

17. Murphy FY, Vesely DL, Jordan RM, et al. Giant invasive prolactinomas. Am J Med. 1987;83(5):995-1002.

18. Yecies D, Ajlan A, Ratliff J, et al. Giant prolactinoma presenting with neck pain and structural compromise of the occipital condyles. J Neurol Surg Rep. 2015;76(2):e297-e301.

19. Zaben MJ, Harrisson SE, Mathad NV. Giant prolactinoma causing cranio-cervical junction instability: a case report. $\mathrm{Br} J$ Neurosurg. 2011;25(6):754-756.

20. Martin MD, Bruner HJ, Wolfla CE, Yoganandan N. Biomechanical implications of extending occipitocervical instrumentation to include the subaxial spine. Neurosurgery. 2010;66(6):1148-1152.

21. Horn EM, Feiz-Erfan I, Lekovic GP, et al. Survivors of occipitoatlantal dislocation injuries: imaging and clinical correlates. J Neurosurg Spine. 2007;6(2):113-120.

22. Kraus DR, Peppelman WC, Agarwal AK, et al. Incidence of subaxial subluxation in patients with generalized rheumatoid arthritis who have had previous occipital cervical fusions. Spine (Phila Pa 1976). 1991;16(suppl 10):S486-S489.

23. Wright NM, Lauryssen C. Vertebral artery injury in C1-2 transarticular screw fixation: results of a survey of the AANS/CNS section on disorders of the spine and peripheral nerves. J Neurosurg. 1998; 88(4):634-640.

24. Puttlitz CM, Melcher RP, Kleinstueck FS, et al. Stability analysis of craniovertebral junction fixation techniques. J Bone Joint Surg Am. 2004;86(3):561-568.

25. Gorek J, Acaroglu E, Berven S, et al. Constructs incorporating intralaminar $\mathrm{C} 2$ screws provide rigid stability for atlantoaxial fixation. Spine (Phila Pa 1976). 2005;30(13):1513-1518.

26. Roberts DA, Doherty BJ, Heggeness MH. Quantitative anatomy of the occiput and the biomechanics of occipital screw fixation. Spine (Phila Pa 1976). 1998;23(10):1100-1108.

27. Winegar CD, Lawrence JP, Friel BC, et al. A systematic review of occipital cervical fusion: techniques and outcomes. J Neurosurg Spine. 2010;13(1):5-16.

28. Nockels RP, Shaffrey $\mathrm{Cl}$, Kanter AS, et al. Occipitocervical fusion with rigid internal fixation: long-term follow-up data in 69 patients. $J$ Neurosurg Spine. 2007;7(2):117-123.

\section{Disclosures}

The authors report no conflict of interest concerning the materials or methods used in this study or the findings specified in this paper. 


\section{Author Contributions}

Conception and design: Chugh, Ray. Acquisition of data: Chugh, Patel, Chua, Arafah, Ray. Analysis and interpretation of data: Chugh, Patel, Arafah. Drafting the article: Chugh, Patel. Critically revising the article: Chugh, Patel, Arafah, Ray. Reviewed submitted version of manuscript: Chugh, Patel, Chua, Arafah. Approved the final version of the manuscript on behalf of all authors: Chugh. Administrative/technical/ material support: Arafah, Ray. Study supervision: Bambakidis, Ray.

\section{Correspondence}

Arunit J. S. Chugh: Case Western Reserve University, University Hospitals Cleveland Medical Center, Cleveland, OH. arunit.chugh@ uhhospitals.org. 